\title{
ARTÍCULOS
}

\section{Crecimiento de renovales de Drimys winteri después de 16 años de aplicados distintos tratamientos de raleo en las cordilleras de Los Andes y de la Costa en Chile}

\author{
Growth of Drimys winteri secondary forest stands following 16 years after thinning \\ in the Andean and Costal Ranges of Chile
}

\author{
René Reyes ${ }^{a *}$, Pablo Donoso ${ }^{\mathrm{b}}$, Claudio Donoso ${ }^{\mathrm{b}}$, Celso Navarro \\ *Autor de correspondencia: aAgrupación de Ingenieros Forestales por el Bosque Nativo, Janequeo 355, Valdivia, Chile, \\ tel.-fax: 56-63-333235, renereyes@ bosquenativo.cl \\ bUniversidad Austral de Chile, Valdivia, Chile. \\ cUniversidad Católica de Temuco, Temuco, Chile.
}

\begin{abstract}
SUMMARY
Secondary Drimys winteri forests are among the most common and productive forests in south-central Chile. However, their management has been scarce or many times inadequate due to lack of silvicultural knowledge. In 1986 and 1990 three field experiments were installed; one in the Coastal range of the province of Ranco (40 $09^{\prime} \mathrm{S}$ ) and two in the Andean range of the province of Llanquihue ( $\left.41^{\circ} 30^{\prime} \mathrm{S}\right)$, to evaluate the effects of different intensities of thinning on the growth and mortality of 28-year-old Drimys winteri secondary forests. After 16 and 10 years of evaluation, great differences were observed among treatments within and between the three experiments. In Hueicoya (Coastal Range) plots thinned at a distance of $3 \mathrm{~m}$ had the highest productivity. In Lenca A (Andean Range) the high mortality rates due to windfalls in the plots with trees spaced at 3 and $4 \mathrm{~m}$ caused the control plots to have the highest productivity rates. In Lenca B (Andean Range) plots thinned at $2.5 \mathrm{~m}$ distance between trees had no significant differences with control plots. At an equal thinning intensity the responses were very different among experiments. We discuss that site quality, residual composition, exposure to winds and rooting characteristics may have influenced these results. In general, it is recommended to thin at 25-45\% residual densities (2-3 m distance between trees at the age of thinning of 9-12 $\mathrm{cm}$ quadratic stand diameter), but always depending on the risk of windfall.
\end{abstract}

Key words: canelo, mortality, thinning, second-growth forest, growth.

\section{RESUMEN}

Los renovales de Drimys winteri son una de las formaciones boscosas más extensas y productivas del centro sur de Chile. Sin embargo, su manejo ha sido escaso y muchas veces inadecuado debido a la falta de conocimiento silvicultural. En 1986 y 1990 se instalaron ensayos para evaluar el efecto de distintas intensidades de raleo en el crecimiento de renovales de $D$. winteri de 28 años de edad en la cordillera de la Costa (Hueicoya, provincia de Ranco, 40 09' S) y en la Cordillera de Los Andes (Lenca A y B, provincia de Llanquihue, $41^{\circ} 30^{\prime}$ S). Después de 16 y 10 años de evaluación, respectivamente, se observaron diferencias entre los tratamientos de cada ensayo y entre ensayos. En Hueicoya con raleo a espaciamiento de $3 \mathrm{~m}$ se presentaron los mejores resultados en productividad. En Lenca A la alta mortalidad producida por el viento con raleo a espaciamientos de 3 y $4 \mathrm{~m}$ hizo que el tratamiento mejor evaluado fuera el testigo. En Lenca B, el raleo a espaciamiento de 2,5 m no produjo diferencias significativas en relación al testigo. A igual intensidad de raleo la respuesta de los renovales en los distintos ensayos fue distinta. Aspectos como calidad de sitio, composición residual, exposición al viento y características del enraizamiento parecen haber influido en este resultado. En general, sería recomendable trabajar con densidades relativas residuales entre 25 y $45 \%$ ( 2 a 3 metros de espaciamiento entre árboles), dependiendo de la vulnerabilidad al viento.

Palabras clave: canelo, mortalidad, intensidad de raleo, efecto del viento, tasa de crecimiento.

\section{INTRODUCCIÓN}

Los renovales de Drimys winteri (J. R. et G. Forster), canelo, constituyen una de las formaciones boscosas más abundantes de la X Región de Chile $\left(40^{\circ}-44^{\circ} \mathrm{S}\right)$, con cerca de 230 mil hectáreas (CONAF et al. 1999). Éstos son muy comunes en áreas cercanas a ciudades, caminos y sectores alterados en general (CONAF et al. 1999). Además, $D$. winteri es una de las especies de mayor potencial de crecimiento del bosque nativo chileno (Navarro et al. 1999). $D$. winteri tiene madera de excelentes propiedades físicas, químicas y mecánicas para la industria papelera (Chesney 
1970) y muy buenas características para la fabricación de tableros de partículas (Urzúa y Poblete 1980), revestimientos interiores, chapas y cajones, tejuelas, vigas y otras piezas en la construcción de casas (Millanao 1984), dada su gran durabilidad y efecto repelente para insectos y otros organismos (Cuevas 1983, Pérez 1983, Niebuhr 1988).

Los renovales de $D$. winteri presentan grandes diferencias de productividad dependiendo del sitio (Calquín 1986). Se han identificado seis clases de sitio para $D$. winteri, las cuales comprenden valores de productividad media entre 1,1 y $16,6 \mathrm{~m}^{3} /$ ha por año, con índices de sitio asociados que varían entre 6,3 y $23,5 \mathrm{~m}$ de altura a una edad clave de 35 años. Las variables drenaje, exposición y pendiente del terreno explican entre 36 y $43 \%$ de la variación en el índice de sitio (Calquín 1986). Estas variables también afectan la composición de especies y las relaciones tamaño-densidad en estos renovales (Donoso et al. 2007). Al menos en el norte de la isla de Chiloé los renovales de D. winteri en exposiciones norte tienden a tener una mayor presencia de especies como Eucryphia cordifolia Cav. y Laureliopsis philippiana (Looser) Schodde, mientras que aquellos en exposiciones sur se asocian a especies como Nothofagus nitida (Phil.) Krasser o mirtáceas. Más allá de la clasificación general desarrollada por Calquín (1986), es muy probable que variaciones topográficas locales tengan importantes efectos en la productividad de renovales de D. winteri.

A pesar de todas las características positivas de D. winteri y su potencial para el manejo silvicultural, las experiencias sobre ensayos silviculturales son escasas. Corvalán et al. (1987) y Garrido (1981) sugieren un primer raleo alrededor de los 10 años de edad. Navarro et al. (1999) han recomendado la implementación de raleos a distintas edades e intensidades dependiendo de la clase de sitio. En clases de sitio I y II sugieren un primer raleo entre los 6 y 8 años de edad, en clases de sitio III y IV entre los 10 y 12 años, y en las clases de sitio V y VI recomiendan un manejo extensivo con intervenciones muy suaves y un primer raleo entre los 20 y 30 años de edad (Calquín 1986, Navarro et al. 1999).

Navarro et al. (1999) reportan los resultados de los primeros años después de haber realizado ensayos de raleo en la Cordillera de la Costa de la provincia de Ranco (Hueicoya). En el presente estudio se reporta un mayor periodo de crecimiento en tales ensayos, así como también en la Cordillera de Los Andes de la provincia de Llanquihue (Lenca), cuyos resultados aún no han sido publicados. En consecuencia, el presente estudio tiene como objetivo evaluar la mortalidad, el crecimiento y la productividad en renovales manejados y no manejados de D. winteri, según la densidad relativa, el área basal residual y la fisiografía, aportando información para futuras pautas de manejo para estos renovales.

\section{MÉTODOS}

Área de estudio. La topografía existente en el área Hueicoya $\left(40^{\circ} 09^{\prime} \mathrm{S}\right)$ es abrupta con pendientes de hasta $80 \%$, predominando las pendientes superiores a 30\%. El ensayo se encuentra en la parte baja de la vertiente occidental de la Cordillera de la Costa a una altitud media de 113 $\mathrm{m}$ s.n.m. Las pendientes en esta zona varían entre $14 \mathrm{y}$ 49\% (Navarro 1993). El clima en esta área corresponde al tipo oceánico templado-húmedo (Cfsb) (Di Castri y Hajek 1976), caracterizándose por precipitaciones altas y temperaturas moderadas debido a la fuerte influencia oceánica. La precipitación media anual cerca del nivel del mar, donde se ubican los renovales estudiados, alcanza alrededor de $3.500 \mathrm{~mm}$. En esta latitud aún existe bastante influencia mediterránea, lo que determina veranos con periodos secos a veces bastante prolongados (Donoso 1993, Rivera et al. 2002). La temperatura media anual es de $9,5^{\circ} \mathrm{C}$, con una media máxima de $17,6^{\circ} \mathrm{C}$ durante febrero y una media mínima de $3,2^{\circ} \mathrm{C}$ durante agosto (Fuenzalida 1965). En invierno los vientos predominantes ${ }^{1}$ provienen del nornoroeste, pudiendo llegar a velocidades máximas entre 110 y $150 \mathrm{~km} / \mathrm{h}$. Los suelos corresponden a la serie Hueicoya, la cual se encuentra al sur del río Valdivia entre los 100 y 1.000 m s.n.m. Son suelos con grandes variaciones de profundidad, predominando los moderadamente profundos, de origen metamórfico y texturas que varían entre moderadamente finas en superficie a finas en profundidad. La materia orgánica es abundante en la superficie pero disminuye bruscamente en profundidad. Se destacan por su bajo contenido de nitrógeno y fósforo y alto contenido de aluminio (Morales et al. 1988).

En el sector norte de Lenca $\left(41^{\circ} 30^{\prime} \mathrm{S}\right.$ ) la topografía es abrupta y montañosa. Al oeste se presenta menos abrupta, existiendo algunas extensiones de terreno con pendientes suaves de aspecto ondulado. En el sector sureste hay pendientes fuertes y numerosas quebradas que confluyen al río Piello (Balharry 1984). El clima en esta área corresponde al tipo oceánico templado-húmedo (Di Castri y Hajek 1976), caracterizándose por altas precipitaciones anuales $(2.000 \mathrm{~mm})$, un tercio de las cuales se presenta durante la temporada estival, lo que lo diferencia consistentemente con el clima de la costa de Hueicoya (Balharry 1984, Donoso 1993). La temperatura media anual es de $11,2^{\circ} \mathrm{C}$, teniendo más de cuatro meses con temperaturas medias mayores a $10^{\circ} \mathrm{C}$ (Fuenzalida 1965). En invierno los vientos predominantes provienen del nornoreste, pudiendo llegar a velocidades máximas entre 90 y $110 \mathrm{~km} / \mathrm{h}$. El enraizamiento de los árboles es superficial, con profundidades que varían entre 10 y $20 \mathrm{~cm}$. La zona donde se establecieron los ensayos corresponde a un área

Dirección Meteorológica de Chile. 2009. Dirección predominante y velocidad máxima del viento medidas en las estaciones meteorológicas de Pichoy (Valdivia) y Tepual (Puerto Montt). Bases de datos referenciales. Santiago, Chile. Consultado 15 mayo 2009. 
que fue talada por los colonos para habilitar terrenos agrícolas, los cuales posteriormente fueron abandonados, surgiendo así el renoval. Existe una presencia abundante de troncos en el piso del bosque, sobre los cuales también han crecido los árboles.

Diseño experimental. El ensayo Hueicoya se estableció entre noviembre de 1985 y febrero de 1986 y está compuesto por 17 parcelas de $600 \mathrm{~m}^{2}(20 \times 30$ metros $)$ dispuestas aleatoriamente. En estas parcelas se aplicaron cinco tratamientos: raleo con distanciamiento de 2 (HT23), 3 (HT3) y 4 metros (HT4), raleo de selección y testigo sin raleo (HTT). En 1996 el tratamiento de raleo HT23 fue intervenido nuevamente para llegar a un distanciamiento de 3 metros. Cada uno de los tratamientos de raleo tuvo tres repeticiones, mientras que el testigo tuvo cinco. El tratamiento raleo de selección no fue considerado en este análisis, debido a que varias de sus parcelas fueron abandonadas a través del tiempo producto de que se borró la pintura de los árboles, impidiendo su identificación.

Los ensayos Lenca A y B se establecieron entre octubre de 1989 y abril de 1990. Lenca A estuvo compuesto por nueve parcelas de $500 \mathrm{~m}^{2}$ ( $25 \times 20$ metros) dispuestas en tres sectores de distinta altitud (tres parcelas por sector), en un área de topografía ondulada a quebrada y un sustrato húmedo pero drenado. En estas parcelas se aplicaron tres tratamientos: raleo a un distanciamiento de 3 (LAT3) y 4 metros (LAT4) y testigo (LATT). Lenca B estuvo compuesto por seis parcelas de $500 \mathrm{~m}^{2}$ dispuestas aleatoriamente en un sector plano con suelo de drenaje lento (cuadro 1). En estas parcelas se aplicaron dos tratamientos: raleo a un distanciamiento de 2,5 metros (LBT25) y testigo (LBTT), con tres repeticiones por tratamiento.

Todos los ensayos se instalaron en renovales de 28 años de edad. El criterio utilizado para llevar a cabo los raleos fue distanciamiento medio y calidad, favoreciendo a individuos dominantes y codominantes.

Cuadro 1. Diseño experimental.

Experimental design.

\begin{tabular}{lcccc}
\hline Ensayo & Parcelas & $\begin{array}{c}\text { Altitud } \\
\text { (m s.n.m.) }\end{array}$ & Exposición & $\begin{array}{c}\text { Pendiente } \\
(\%)\end{array}$ \\
\hline Hueicoya* & $1-18$ & $25-180$ & S-SO & $15-49$ \\
Lenca A** & $1-9$ & $100-500$ & $\begin{array}{c}\text { Plano, S-SE, } \\
\text { S-SO }\end{array}$ & $0-45$ \\
& & & Plano & 0 \\
\hline
\end{tabular}

* Parcelas 5-9-17 raleo $2 \mathrm{~m}, 2-4-11$ raleo $3 \mathrm{~m}, 1-10-12$ raleo $4 \mathrm{~m}$, 3-8-16 raleo de selección, y 6-7-14-15-18 testigos.

** Parcelas 1-6-7 raleo 3 m, 2-5-9 raleo 4 m, y 3-4-8 testigos. Parcelas 11-12-16 raleo 2,5 m, y 13-14-15 testigos.

El ensayo en Hueicoya fue evaluado en 1986, 1990, 1996 y 2002, y aquellos ubicados en Lenca A y B en 1991, 1996 y 2001. Las variables medidas en cada una de estas oportunidades han sido especie, diámetro a la altura del pecho (DAP), sanidad, forma, posición sociológica, calidad y altura total. En la medición realizada en Lenca A el año 2001 se midió además la profundidad de arraigamiento de los árboles que fueron desraizados por el viento. El análisis comparativo entre ensayos y tratamientos se realizó para la variación en número de árboles (mortalidad), el diámetro medio cuadrático (DMC), el área basal, la altura total y el volumen, aplicando análisis de varianza (ANDEVA) y la prueba de Tukey $(P<0,05)$ para todo el periodo de evaluación.

Para la estimación de altura y volumen se utilizaron las funciones mencionadas en el cuadro 2. Por otra parte, la mortalidad y el diámetro medio cuadrático se calcularon con base en las siguientes fórmulas:

Mortalidad:

$$
M=\left(\sqrt[n]{\left(\frac{N f}{N i}-1\right)}\right) * 100
$$

Diámetro medio cuadrático $(\mathrm{cm})$ :

$$
D M C=\sqrt[2]{\frac{A B * 40000}{N * 3,1416}}
$$

Donde,

M: tasa de mortalidad (\%); n: número de años entre el inicio y el final del periodo; Nf: número de árboles al final del periodo; Ni: número de árboles al inicio del periodo; $\mathrm{AB}$ : área basal por hectárea $\left(\mathrm{m}^{2}\right) ; \mathrm{N}$ : número de árboles por hectárea.

Características de los renovales antes de la aplicación de los tratamientos. En Hueicoya los renovales estudiados correspondían a bosques casi puros, donde $D$. winteri representó un $81 \%$ de los individuos. El $19 \%$ restante estaba compuesto principalmente por Amomyrtus luma (Molina) Legrand et Kausel. En Lenca A $70 \%$ de los árboles correspondía a $D$. winteri, $9 \%$ a $N$. nítida y $21 \%$ a otras especies (A. luma, L. philippiana, Embothrium coccineum Forst. et Forst.). En Lenca B, en cambio, $57 \%$ correspondió a $D$. winteri, $23 \%$ a N. nitida y $20 \%$ a otras especies (A. luma, E. coccineum, L. philippiana, Weinmannia trichosperma Cav., Caldcluvia paniculata (Cav.) D. Don, Lomatia ferruginea (Cav.) R. Br., y otras).

A partir de los datos originales de las parcelas, a los 28 años el crecimiento medio de los renovales (sin considerar mortalidad) en Hueicoya era de $12,8 \mathrm{~m}^{3} / \mathrm{ha}$ por año (10,3-16,2 $\mathrm{m}^{3} / \mathrm{ha}$ por año), en Lenca A de $14,2 \mathrm{~m}^{3} / \mathrm{ha}$ por año $\left(10,7-19,4 \mathrm{~m}^{3} / \mathrm{ha}\right.$ por año), y en Lenca B de $11,1 \mathrm{~m}^{3} / \mathrm{ha}$ por año $\left(8,7-14,6 \mathrm{~m}^{3} / \mathrm{ha}\right.$ por año) (cuadro 3) (Navarro 1993). Si se mantienen estos valores de productividad hasta la edad de 35 años, Lenca A equivaldría a una clase de sitio II, y Lenca B y Hueicoya a una clase de sitio III (Calquín 1986). 
Cuadro 2. Funciones de altura y volumen empleadas en los ensayos de raleo de renovales de D. winteri en Hueicoya y Lenca. Height and volume functions used in D. winteri secondary forests in Hueicoya and Lenca.

\begin{tabular}{lccc}
\hline \multicolumn{1}{c}{ Especie } & Tipo de función / sitio & Funciones & Fuente \\
\hline Drimys winteri & Altura / Lenca & $e^{3,0831364-1,6289897 * D A P^{-0,5}}$ & Corvalán et al. 1987 \\
& Altura / Hueicoya & $9,252717+0,2523175 * D A P$ & Gunckel 1980 \\
& Volumen / Lenca & $(-0,5188637+0,3191276 * H) *(D A P / 100)^{2}$ & Niebuhr 1988 \\
& Volumen / Hueicoya & $0,01222+0,0000315502 *\left(D A P^{2}\right) * H$ & Gunckel 1980 \\
Nothofagus nitida & Volumen / todos & $\left(0,0214+0,6205 *\left(\frac{D A P}{100}\right)^{2} *(-7,1755+0,9909 * H)\right.$ & Corvalán et al. 1987 \\
Amomyrtus luma & Volumen / todos & $\left(0,0381+0,4731 *\left(\frac{D A P}{100}\right)^{2} *(-5,0851+0,7704 * H)\right.$ & Corvalán et al. 1987 \\
Embothrium coccineum & Volumen / todos & $\left(\frac{D A P}{100}\right)^{2} *(4,1675+0,1303 *(-6,3217+0,8706 * H))$ & Corvalán et al. 1987 \\
Laureliopsis philippiana & Volumen / todos & $\left(\frac{D A P}{100}\right)^{2} *(-0,7363118+0,33301 * H)$ & Corvalán et al. 1987 \\
Eucryphia cordifolia & Volumen / todos & $\left(\frac{D A P}{100}\right)^{2} *(1,7972+0,3529 *(-0,1895+0,6633 * H))$ & Corvalán et al. 1987 \\
\hline
\end{tabular}

Cuadro 3. Principales variables de rodal de cada ensayo antes de los raleos.

Main stand variables in each experiment before thinning.

\begin{tabular}{|c|c|c|c|c|}
\hline \multirow{2}{*}{ Parámetros* } & & \multicolumn{3}{|c|}{ Ensayo } \\
\hline & & Hueicoya** & Lenca A & Lenca B \\
\hline \multirow{4}{*}{$\begin{array}{l}\text { Número de árboles } \\
\text { (N/ha) }\end{array}$} & Med. & 6.134 & 6.451 & 8.083 \\
\hline & $S$ & 1243 & 1274 & 1.048 \\
\hline & Máx. & 8.451 & 8.280 & 9.960 \\
\hline & Mín. & 3.784 & 4.160 & 6.940 \\
\hline \multirow{4}{*}{ Área basal (m²/ha) } & Med. & 56,6 & 68,8 & 53,4 \\
\hline & S & 8,6 & 6,6 & 6,3 \\
\hline & Máx. & 74,2 & 78,2 & 62,6 \\
\hline & Mín. & 44,5 & 55,4 & 46,9 \\
\hline \multirow{4}{*}{ Volumen $\left(\mathrm{m}^{3} / \mathrm{ha}\right)$} & Med. & 360 & 397 & 309 \\
\hline & S & 49,5 & 53,6 & 68,9 \\
\hline & Máx. & 454 & 466 & 410 \\
\hline & Mín. & 288 & 315 & 244 \\
\hline Altura total (m) & Med. & 12,6 & 12,3 & 9,7 \\
\hline DMC (cm) & - & 11 & 12 & 9 \\
\hline Edad (años) & Med. & 28 & 29 & 29 \\
\hline $\begin{array}{l}\text { Incremento }\left(\mathrm{m}^{3} / \mathrm{ha}\right. \\
\text { por año })^{* *}\end{array}$ & Med. & 12,8 & 14,2 & 11,1 \\
\hline
\end{tabular}

* Med.: media; S: desviación estándar; Máx.: máximo; Mín.: mínimo. DMC: diámetro medio cuadrático.

** Navarro (1993).
La distribución diamétrica de todos los sectores al momento de establecer los ensayos era del tipo exponencial negativa. Esta situación refleja una fuerte competencia entre los individuos de $D$. winteri, lo que provoca la supresión de un gran número de ellos. De esta forma una proporción importante de los árboles se concentraba en la clase diamétrica de 5 a $10 \mathrm{~cm}$ (Hueicoya 52,5\%, Lenca A $57,7 \%$ y Lenca B 78,5\%) (Navarro 1993). Con base en las funciones publicadas por Donoso et al. (2007), la densidad relativa promedio de los renovales en Hueicoya era de $84 \%$, en Lenca A de un $104 \%$, y en Lenca B de $77 \%$, es decir, eran renovales densos o muy densos, con densidades relativas que ilustran que debe haber habido en esos momentos una intensa mortalidad por competencia.

Características de los renovales después de los raleos. Las intervenciones realizadas correspondieron a raleos por lo bajo que afectaron considerablemente a especies tolerantes como A. luma y L. philippiana. En HT3 y HT4 la corta también afectó a individuos dominantes y codominantes, la mayor parte de $D$. winteri y $N$. nitida, debido a la gran intensidad de la intervención. Estos raleos aumentaron el DMC, generando curvas de distribución diamétrica con forma de campana, salvo en HT23 y LBT25, donde se mantuvo la forma exponencial negativa.

En Hueicoya se extrajo 90, 80 y $64 \%$ de los árboles y 77, 64 y 39\% del área basal en los tratamientos HT4, HT3 y HT23, respectivamente, pudiendo calificarse los dos primeros como raleos muy fuertes o grado $\mathrm{D}$, y el tercero como raleo fuerte o grado C (Nyland 2002). En los tratamientos LAT3 y LAT4 se extrajo 80 y $85 \%$ de los árboles y 60 y $69 \%$ del área basal, respectivamente, pudiendo calificarse como raleos grado D. En LBT25 se 
Cuadro 4. Intensidad de extracción de los raleos efectuados en cada tratamiento en Hueicoya y Lenca. Thinning intensity in each treatment and experiment.

\begin{tabular}{lcccccccccccc}
\hline \multirow{2}{*}{ Ensayo } & $\begin{array}{c}\text { Tratamiento } \\
\text { de raleo }\end{array}$ & \multicolumn{3}{c}{ Antes de la intervención } & \multicolumn{3}{c}{ Después de la intervención } & \multicolumn{3}{c}{ Extracción (\%) } \\
\cline { 3 - 13 } & N/ha & AB/ha & V/ha & DR* & N/ha & AB/ha & V/ha & DR* & N/ha & AB/ha & V/ha \\
\hline Hueicoya & HT4 & 5.367 & 47,8 & 309,1 & 70 & 611 & 11,0 & 67,5 & 15 & 89 & 77 & 78 \\
(1986) & HT3 & 5.682 & 56,6 & 360,9 & 82 & 1.095 & 20,4 & 126,6 & 28 & 81 & 64 & 65 \\
& HT23 & 7.001 & 62,4 & 395,8 & 91 & 2.495 & 36,5 & 216,7 & 51 & 64 & 39 & 45 \\
& HTT & 6.772 & 60,6 & 372,2 & 88 & 6.772 & 60,6 & 372,2 & 88 & 0 & 0 & 0 \\
\hline Lenca A & LAT4 & 6.093 & 68,0 & 365,5 & 97 & 920 & 21,1 & 112,2 & 28 & 85 & 69 & 69 \\
(1991) & LAT3 & 6.613 & 69,3 & 392,9 & 100 & 1.327 & 27,8 & 156,4 & 38 & 80 & 60 & 60 \\
& LATT & 6.451 & 68,8 & 434,0 & 99 & 6.451 & 68,8 & 434,0 & 99 & 0 & 0 & 0 \\
\hline \multirow{2}{*}{ Lenca B B (1991) } & LBT25 & 8.587 & 55,6 & 294,2 & 83 & 1.827 & 21,4 & 100,1 & 30 & 79 & 62 & 66 \\
& LBTT & 8.083 & 53,4 & 324,1 & 80 & 8.083 & 53,4 & 324,1 & 80 & 0 & 0 & 0 \\
\hline
\end{tabular}

* Donoso et al. (2007).

Cuadro 5. Variación del número de árboles en los diferentes tratamientos de raleo de renovales de D. winteri en Hueicoya y Lenca. Variation in number of trees in the different thinning treatments in D. winteri secondary forests in Hueicoya and Lenca.

\begin{tabular}{lccccccccc}
\hline \multirow{2}{*}{ Ensayo } & \multirow{2}{*}{ Tratamiento } & \multicolumn{7}{c}{ Número de árboles por hectárea } & \multicolumn{5}{c}{ Tasa anual de mortalidad (\%) } \\
\cline { 3 - 10 } & & 1986 & $1990^{(1)}$ & 1996 & $2001^{(2)}$ & $86-90$ & $90-96$ & $96-01$ & Media \\
\hline \multirow{4}{*}{ Hueicoya } & HT4 & 611 & 573 & 534 & 500 & 1,6 & 1,1 & 1,0 & 1,1 \\
& HT3 & 1.094 & 933 & 867 & 828 & 3,6 & 1,3 & 0,6 & 1,9 \\
& HT23 ${ }^{(3)}$ & 2.495 & 2.433 & 2.227 & - & 0,6 & 1,4 & - & 1,1 \\
& & - & - & 1.139 & 929 & - & - & 3,0 & - \\
& HTT & 6.772 & 6.226 & 5.108 & 3.925 & 1,6 & 3,3 & 3,9 & $2,9^{*}$ \\
\hline \multirow{2}{*}{ Lenca A } & LAT4 & - & 920 & 660 & 553 & - & 6,4 & 3,5 & 5,0 \\
& LAT3 & - & 1.327 & 1.013 & 867 & & 5,3 & 3,1 & 4,2 \\
& LATT & - & 6.010 & 4.740 & 3.840 & & 4,9 & 4,3 & 4,6 \\
\hline Lenca B & LBT25 & - & 1.827 & 1.600 & 1.460 & - & 2,6 & 1,8 & 2,2 \\
& LBTT & - & 7.580 & 6.507 & 5.353 & - & 3,0 & 3,8 & 3,4 \\
\hline
\end{tabular}

1. Mediciones realizadas el invierno de 1990 en Hueicoya y de 1991 en Lenca.

2. Mediciones realizadas en primavera de 2001 en Lenca y durante la primavera de 2002 en Hueicoya.

3. En 1996 se volvieron a ralear las parcelas de $2 \times 2 \mathrm{~m}$ dejándolas a un distanciamiento final de 3x3 $\mathrm{m}$.

* Testigo con mortalidad mayor que rodales raleados $(P<0,05)$. En las demás comparaciones no hubo diferencias significativas.

extrajo el $78 \%$ de los árboles y el $61 \%$ del área basal, pudiendo calificarse también como un raleo grado D (cuadro 4). Las distribuciones originales y posraleo de las parcelas en Hueicoya son reportadas por Navarro et al. (1999).

\section{RESULTADOS}

Mortalidad. En Hueicoya las tasas anuales de mortalidad de las parcelas donde se aplicaron tratamientos de raleo fueron bajas. Algunas excepciones se observaron en HT3 y HT23 (cuadro 5). En el primer caso, durante el periodo 1986-1990 la tasa de mortalidad fue de 3,6\% anual, valor muy superior al de los otros tratamientos $(0,6$ a $1,6 \%)$. Esta mortalidad se produjo debido a la formación de callejones en algunas parcelas durante la faena de raleo, los cuales ocasionaron la caída de una gran cantidad de árboles (Navarro 1993). En el segundo caso, la aplicación del raleo a 3 metros en 1996 en aquellas parcelas raleadas inicialmente a 2 metros causó un aumento fuerte de la mortalidad, elevando la tasa anual de 1,1\% (1986-1996) a 3,0\% (1996-2002). En ese periodo el tratamiento HTT tuvo una tasa anual de mortalidad de $3,9 \%$. A pesar de lo anterior, sólo hubo diferencias significativas entre las tasas de mortalidad de los tratamientos de raleo y el tratamiento testigo, considerando los 16 años de evaluación $(P=0,046)$.

Los tratamientos LAT3 y LAT4 experimentaron una fuerte mortalidad durante todo el periodo de evaluación, muy especialmente en los primeros cinco años $(5,3$ y $6,4 \%$ anual). El análisis estadístico no mostró diferencias significativas entre las tasas anuales de mortalidad de ambos tratamientos y el testigo (cuadro 5) $(P=0,715)$. 
En Lenca B el tratamiento LBT25 tuvo una tasa anual de mortalidad de 2,6\% durante los primeros cinco años de evaluación, la cual fue muy similar a la del tratamiento LBTT $(3,0 \%)$. Sin embargo, después disminuyó considerablemente $(1,8 \%)$, diferenciándose de LBTT $(3,8 \%)$ (cuadro 5), a pesar de lo cual las tasas de mortalidad anual de ambos tratamientos no tuvieron diferencias estadísticamente significativas considerando los 10 años de evaluación $(P=0,088)$.

Crecimiento en diámetro. En todos los ensayos los árboles de las parcelas raleadas tuvieron crecimientos significativamente superiores que aquellos de las parcelas testigo (cuadro 6). En Hueicoya el crecimiento promedio de los tratamientos HT3 y HT4 (0,52 y 0,55 $\mathrm{cm} / \mathrm{año}$ ) fue significativamente superior a aquel de los tratamientos HT23 y HTT $(P=0,001)$, a pesar de que el crecimiento en el tratamiento HT23, durante el periodo 1996-2002, fue similar a aquel de los raleos más fuertes. De la misma manera, en Lenca A el crecimiento de los tratamientos de raleo fue significativamente mayor que en las parcelas testigo $(P=0,044)$. En Lenca B también fueron significativamente diferentes los crecimientos entre LBT25 y LBTT $(P=0,036)$.

Crecimiento en área basal. En HT4 y HT3 se redujo la densidad relativa de los renovales de 70 y $82 \%$ inicial a 15 y $28 \%$, respectivamente. Durante todo el periodo de evaluación la tasa de incremento anual en área basal fue de 0,7 y $1,0 \mathrm{~m}^{2} /$ ha por año, respectivamente, muy superior $(P<0,05)$ a HTT $\left(0,3 \mathrm{~m}^{2} /\right.$ ha por año) (cuadro 7$)$. El tratamiento HT23 redujo la densidad relativa de los renovales de $91 \%$ a $51 \%$. Al aplicar el raleo a 3 metros en 1996 se redujo nuevamente la densidad relativa de $56 \%$ a $42 \%$. En el periodo en que HT23 estuvo raleado a 2 metros el incremento anual en área basal fue de $1,0 \mathrm{~m}^{2} /$ ha por año, significativamente superior a HTT $(P<0,05)$. Posteriormente, disminuyó a $0,4 \mathrm{~m}^{2} /$ ha por año. En este periodo HT23 no presentó diferencias estadísticamente significativas con el tratamiento testigo (cuadro 7) $(P=0,22)$. Considerando todo el periodo de evaluación, HT23 fue superior a HTT $(P<0,05)$.

En LAT4 y LAT3 se redujo la densidad relativa de los renovales de 97 y $100 \%$ inicial a 28 y $38 \%$, respectivamente. Durante todo el periodo de evaluación su incremento en área basal fue de 0,56 y $0,61 \mathrm{~m}^{2} /$ ha por año, respectivamente, muy similar al incremento de LATT $\left(0,6 \mathrm{~m}^{2} / \mathrm{ha}\right.$ por año) (cuadro 7).

En LBT25 se redujo la densidad relativa del renoval de $83 \%$ a $30 \%$. Durante el periodo 1991-1996 LBT25 y LBTT tuvieron el mismo incremento anual en área basal; sin embargo, en el periodo 1996-2001 el crecimiento fue casi el doble en LBT25 (cuadro 7). Considerando todo el periodo de evaluación hubo diferencias significativas entre ambos tratamientos $(P=0,048)$.

Crecimiento en volumen. En Hueicoya el volumen promedio antes de la aplicación de los tratamientos era de $360 \mathrm{~m}^{3} / \mathrm{ha}$. En HT4 y HT3 se redujo el volumen a 68 y $127 \mathrm{~m}^{3} / \mathrm{ha}$, respectivamente. Durante el periodo de evaluación el incremento volumétrico de HT4, HT3 y HTT fue de 5,2, 7,0 y $1,8 \mathrm{~m}^{3} /$ ha por año, respectivamente. El tratamiento HT23 redujo el volumen del renoval a $217 \mathrm{~m}^{3} / \mathrm{ha}$. Mientras estuvo raleado a 2 metros, su incremento volumétrico fue de 6,0 $\mathrm{m}^{3} /$ ha por año, el cual fue mayor al de HTT $\left(4,1 \mathrm{~m}^{3} /\right.$ ha por año) y HT4 (5,0 m³/ha por año). En 1996 se aplicó el raleo 3 metros, en el cual se cosecharon $88 \mathrm{~m}^{3} / \mathrm{ha}$. Entre 1996 y 2002 el incremento volumétrico de este tratamiento fue de $5,2 \mathrm{~m}^{3} /$ ha por año, el cual fue levemente inferior a los 5,4 y $7,4 \mathrm{~m}^{3} /$ ha por año que en ese periodo tuvieron HT4 y HT3. Sólo hubo diferencias estadísticamente significativas entre los tratamientos de raleo y el tratamiento testigo $(P=0,032)$ (cuadro 8).

En Lenca A el volumen inicial era de $380 \mathrm{~m}^{3} / \mathrm{ha}$. En LAT4 y LAT3 se redujo este volumen a 112 y $156 \mathrm{~m}^{3} / \mathrm{ha}$, respectivamente. El incremento volumétrico neto durante el

Cuadro 6. Variación del diámetro medio cuadrático en los ensayos de raleo de renovales de $D$. winteri en Hueicoya y Lenca. Variation in quadratic stand diameter in the Hueicoya and Lenca thinning experiments in D. Winteri secondary forests.

\begin{tabular}{|c|c|c|c|c|c|c|c|c|c|}
\hline \multirow{2}{*}{ Ensayo } & \multirow{2}{*}{$\begin{array}{c}\text { Tratamiento } \\
\text { de raleo }\end{array}$} & \multicolumn{4}{|c|}{ Diámetro medio cuadrático $(\mathrm{cm})$} & \multicolumn{4}{|c|}{ Crecimiento anual periódico (cm/año) } \\
\hline & & 1986 & 1990 & 1996 & 2001 & $86-90$ & $90-96$ & $96-01$ & Media* \\
\hline \multirow[t]{5}{*}{ Hueicoya } & HT4 & 15,1 & 18,2 & 20,9 & 23,9 & 0,78 & 0,45 & 0,50 & $0,55 \mathrm{a}$ \\
\hline & HT3 & 15,3 & 18,0 & 21,2 & 23,6 & 0,68 & 0,53 & 0,40 & $0,52 \mathrm{a}$ \\
\hline & HT23 & 13,6 & 14,6 & 16,3 & - & 0,25 & 0,28 & - & $0,27 b$ \\
\hline & & - & - & 19,0 & 21,8 & - & - & 0,47 & - \\
\hline & HTT & 10,7 & 11,7 & 13,0 & 14,5 & 0,25 & 0,22 & 0,25 & $0,24 b$ \\
\hline \multirow[t]{3}{*}{ Lenca A } & LAT4 & - & 17,1 & 22,1 & 24,8 & - & 0,83 & 0,54 & $0,77 \mathrm{a}$ \\
\hline & LAT3 & - & 16,3 & 20,5 & 22,3 & - & 0,69 & 0,37 & $0,60 \mathrm{a}$ \\
\hline & LATT & - & 12,0 & 14,8 & 16,7 & - & 0,56 & 0,38 & $0,47 \mathrm{~b}$ \\
\hline \multirow[t]{2}{*}{ Lenca B } & LBT25 & - & 12,2 & 16,0 & 18,4 & - & 0,76 & 0,48 & $0,62 \mathrm{a}$ \\
\hline & LBTT & - & 9,3 & 11,0 & 12,5 & - & 0,34 & 0,30 & $0,32 b$ \\
\hline
\end{tabular}

*Letras distintas indican medias significativamente diferentes $(P<0,05)$ en cada ensayo. 
Cuadro 7. Variación del área basal en los ensayos de raleo de renovales de D. winteri en Hueicoya y Lenca.

Variation in basal area in the Hueicoya and Lenca thinning experiments in D. Winteri secondary forests.

\begin{tabular}{|c|c|c|c|c|c|c|c|c|c|c|c|}
\hline \multirow[t]{2}{*}{ Ensayo } & \multirow{2}{*}{$\begin{array}{l}\text { Tratamiento } \\
\text { de raleo }\end{array}$} & \multirow[t]{2}{*}{ Parcela } & \multicolumn{4}{|c|}{ Área basal $\left(\mathrm{m}^{2} / \mathrm{ha}\right)$} & \multicolumn{4}{|c|}{$\begin{array}{l}\text { Crecimiento anual periódico } \\
\left(\mathrm{m}^{2} / \text { ha por año }\right)\end{array}$} & \multirow{2}{*}{$\begin{array}{c}\% \\
\text { aumento }\end{array}$} \\
\hline & & & 1986 & $1990 / 91$ & 1996 & $2001 / 02$ & $86-90$ & $90-96$ & 96-01 & Media* & \\
\hline \multirow[t]{5}{*}{ Hueicoya } & HT4 & & 11,0 & 14,4 & 18,4 & 22,7 & 0,9 & 0,7 & 0,7 & $0,7 \mathrm{a}$ & 106 \\
\hline & HT3 & & 20,4 & 22,7 & 30,5 & 36,3 & 0,6 & 1,3 & 1,0 & $1,0 \mathrm{a}$ & 78 \\
\hline & HT23 & & 36,5 & 39,2 & 46,6 & - & 0,7 & 1,2 & - & - & - \\
\hline & & & - & - & 32,2 & 34,7 & - & - & 0,4 & $0,8 \mathrm{a}$ & - \\
\hline & HTT & & 60,6 & 65,7 & 67,9 & 65,1 & 1,3 & 0,4 & $-0,5$ & $0,3 b$ & 7 \\
\hline \multirow[t]{12}{*}{ Lenca A } & LAT4 & 2 & - & 21,0 & 25,1 & 27,0 & - & 0,8 & 0,4 & 0,6 & 29 \\
\hline & & 5 & - & 22,1 & 17,2 & 18,2 & - & $-1,0$ & 0,2 & $-0,4$ & -18 \\
\hline & & 9 & - & 20,2 & 33,6 & 35,0 & - & 2,7 & 0,3 & 1,5 & 73 \\
\hline & & Media & & 21,1 & 25,3 & 26,7 & - & - & - & $0,6 \mathrm{a}$ & - \\
\hline & LAT3 & 1 & - & 26,3 & 38,1 & 37,9 & - & 2,3 & 0,0 & 1,2 & 44 \\
\hline & & 6 & - & 26,9 & 24,8 & 28,0 & - & $-0,4$ & 0,6 & 0,1 & 4 \\
\hline & & 7 & - & 26,8 & 37,1 & 35,7 & - & 2,1 & $-0,3$ & 0,9 & 33 \\
\hline & & Media & & 26,7 & 33,3 & 33,9 & - & - & - & $0,7 \mathrm{a}$ & - \\
\hline & LATT & 3 & - & 72,6 & 86,6 & 87,9 & - & 2,8 & 0,3 & 1,5 & 21 \\
\hline & & 4 & - & 62,4 & 75,6 & 81,1 & - & 2,6 & 1,1 & 1,9 & 30 \\
\hline & & 8 & - & 71,9 & 66,7 & 56,4 & - & $-1,0$ & $-2,1$ & $-1,6$ & -22 \\
\hline & & Media & & 69,0 & 76,3 & 75,1 & - & - & - & $0,6 \mathrm{a}$ & - \\
\hline \multirow[t]{2}{*}{ Lenca B } & LBT25 & & - & 21,4 & 32.3 & 38,8 & - & 2,2 & 1,3 & $1,7 \mathrm{a}$ & 81 \\
\hline & LBTT & & - & 51,2 & 62,3 & 66,0 & - & 2,2 & 0,7 & $1,5 \mathrm{a}$ & 29 \\
\hline
\end{tabular}

* Letras distintas indican medias significativamente diferentes $(P<0,05)$ en cada ensayo.

Cuadro 8. Incremento bruto y neto del volumen en los ensayos de raleo de renovales de D. winteri en Hueicoya y Lenca. Gross and net volume growth in the Hueicoya and Lenca thinning experiments in D. Winteri secondary forests.

\begin{tabular}{|c|c|c|c|c|c|c|c|c|c|c|c|c|c|c|}
\hline \multirow[t]{2}{*}{ Ensayo } & \multirow{2}{*}{$\begin{array}{l}\text { Tratamiento } \\
\text { de raleo }\end{array}$} & \multirow{2}{*}{$\begin{array}{c}\begin{array}{c}\text { Incremento } \\
\text { bruto }\end{array} \\
\mathrm{m}^{3} / \mathrm{ha}\end{array}$} & \multicolumn{2}{|c|}{ Mortalidad } & \multicolumn{2}{|c|}{ Incremento neto } & \multicolumn{4}{|c|}{ Volumen neto $\left(\mathrm{m}^{3} / \mathrm{ha}\right)$} & \multicolumn{4}{|c|}{$\begin{array}{l}\text { Incremento neto en volumen } \\
\qquad\left(\mathrm{m}^{3} / \mathrm{ha} \text { por año }\right)\end{array}$} \\
\hline & & & $\mathrm{m}^{3} / \mathrm{ha}$ & $\%$ & $\mathrm{~m}^{3} / \mathrm{ha}$ & $\%$ & 1986 & 1990 & 1996 & 2001 & $86-90$ & $90-96$ & $96-01$ & Media $^{(3)}$ \\
\hline \multirow[t]{6}{*}{ Hueicoya } & HT4 & 93,8 & 11,1 & 11,8 & 82,7 & 88,2 & 67,5 & 89,1 & 117,8 & 150,2 & 5,4 & 4,8 & 5,4 & $5,2 \mathrm{a}$ \\
\hline & HT3 & 140,7 & 29,1 & 20,7 & 111,6 & 79,3 & 126,6 & 145,0 & 193,9 & 238,2 & 4,6 & 8,1 & 7,4 & $7,0 \mathrm{a}$ \\
\hline & HT23 & 165,6 & 74,1 & 44,7 & 91,5 & 55,3 & - & - & - & - & - & - & - & - \\
\hline & $2 \mathrm{~m}^{(1)}$ & 82,7 & 22,6 & - & 60,1 & - & 216,7 & 254,6 & 276,8 & - & 9,5 & 3,7 & - & - \\
\hline & $3 \mathrm{~m}^{(2)}$ & 82,9 & 51,5 & - & 31,4 & - & - & - & 189,3 & 220,7 & - & - & 5,2 & $5,7 \mathrm{a}$ \\
\hline & HTT & 172,4 & 137,1 & 79,5 & 35,3 & 20,5 & 372,2 & 404,8 & 413,4 & 400,2 & 8,2 & 1,4 & $-2,2$ & $1,8 \mathrm{~b}$ \\
\hline \multirow[t]{3}{*}{ Lenca A } & LAT4 & 113,3 & 45,9 & 40,5 & 67,4 & 59,5 & - & 112,2 & 146,2 & 157,5 & - & 6,8 & 2,2 & $4,5 \mathrm{a}$ \\
\hline & LAT3 & 143,3 & 59,2 & 41,3 & 84,1 & 58,7 & - & 156,4 & 194,8 & 204,0 & - & 7,7 & 1,8 & $4,8 \mathrm{a}$ \\
\hline & LATT & 221,1 & 87,2 & 39,4 & 133,9 & 60,6 & - & 404,0 & 504,0 & 537,9 & - & 20,0 & 6,8 & $13,4 \mathrm{a}$ \\
\hline \multirow[t]{2}{*}{ Lenca B } & LBT25 & 167,1 & 24,5 & 14,7 & 142,6 & 85,3 & - & 100,1 & 170,4 & 207,4 & - & 14,1 & 7,4 & $10,7 \mathrm{a}$ \\
\hline & LBTT & 202,5 & 80,7 & 39,9 & 121,8 & 60,1 & - & 324,1 & 406,6 & 445,9 & - & 16,5 & 7,9 & $12,2 \mathrm{a}$ \\
\hline
\end{tabular}

(1) Durante el periodo 1986-1996. (2) Durante el periodo 1996-2002. (3) Letras distintas indican medias significativamente diferentes. $(P<0,05)$ en cada ensayo.

periodo de evaluación fue de 4,5 y $4,8 \mathrm{~m}^{3} / \mathrm{ha}$ por año. El incremento de LATT, en cambio, fue de $13,4 \mathrm{~m}^{3} /$ ha por año. A pesar de las diferencias, éstas no fueron estadísticamente significativas entre los tratamientos $(P=0,67)$.
En Lenca B el volumen inicial era de $309 \mathrm{~m}^{3} / \mathrm{ha}$. En LBT25 se redujo el volumen a $100 \mathrm{~m}^{3} / \mathrm{ha}$. Las tasas de crecimiento de LBT25 y LBTT fueron de 10,7 y $12,2 \mathrm{~m}^{3} / \mathrm{ha}$ por año. No hubo diferencias estadísticamente significativas entre ambos tratamientos $(P=0,71)$. 


\section{DISCUSIÓN}

Renovales no manejados. El desarrollo de las parcelas testigo en los tres ensayos es distinto, siendo explicado por dos aspectos fundamentales: calidad de sitio y composición de especies. El primer aspecto genera una diferencia importante en términos de crecimiento en altura y velocidad en el desarrollo de los árboles, lo cual favorece especialmente a los renovales ubicados en Lenca A. Esto redunda en un mayor volumen, dado un mismo diámetro medio, y en mayores tasas de crecimiento (Calquín 1986). El segundo aspecto determina la ocupación del sitio (Oliver y Larson 1990, Nyland 2002). En Hueicoya el renoval es prácticamente monoespecífico, lo cual lo hace menos eficiente en la ocupación del sitio en comparación a los renovales ubicados en Lenca, donde una parte importante de los árboles son especies tolerantes como L. philippiana o intolerantes como N. nitida. Considerando que las especies acompañantes de D. winteri tienen características de tolerancia, fenológicas y morfológicas distintas, en los rodales mixtos se espera que haya mayor complementariedad en el uso de los recursos y por ello mayor productividad (Kelty 1992). A los 38 años de edad el renoval de Lenca A acumuló $85 \mathrm{~m}^{2} / \mathrm{ha}$ y continúa creciendo; en cambio, en Hueicoya se estancó cerca de los $68 \mathrm{~m}^{2} /$ ha (figura 1 ).

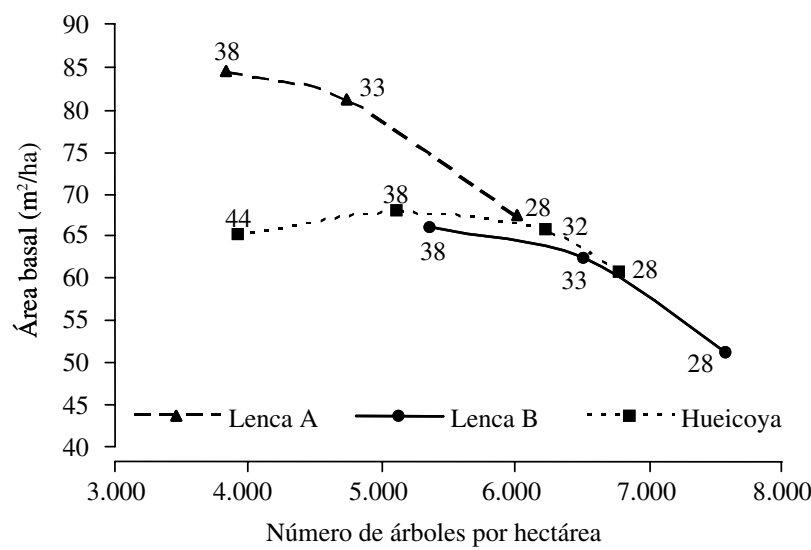

Figura 1. Curvas de densidad máxima a partir de parcelas testigo. Los números sobre las curvas representan la edad (años) del renoval.

Maximum density curves based on control plots.

Si bien Hueicoya es un sitio de mejor calidad que Lenca $\mathrm{B}$, se sugiere que la composición pura del renoval $(80 \% \mathrm{D}$. winteri) puede haber limitado la ocupación del sitio y, por tanto, la acumulación de área basal y volumen, en contraste con Lenca B, donde una mayor diversidad arbórea habría permitido una mejor ocupación de los distintos nichos del sitio (Nyland 2002, Oliver y Larson 1990). Es importante aclarar que este nivel máximo de ocupación de sitio está referido sólo a aquellos árboles que fueron medidos desde que se instaló el ensayo. Durante el transcurso de los años se han reclutado nuevos individuos sobre el umbral de medición $(5 \mathrm{~cm}$, ingrowth), principalmente de especies tolerantes y semitolerantes, las cuales comienzan a reemplazar paulatinamente a $D$. winteri adicionando área basal y volumen (Appel 1993). Este componente del crecimiento del rodal no fue considerado. Soto y Donoso (2006) señalan que el proceso de reclutamiento de especies tolerantes en renovales de $D$. winteri ocurre particularmente luego de que los individuos de $D$. winteri alcanzan aproximadamente $12 \mathrm{~cm}$ de diámetro o $15 \mathrm{~m}$ de altura.

Renovales manejados. En Lenca A la mortalidad en los tratamientos LAT3 y LAT4, debido al daño por viento, alteró la estructura del bosque y mermó su productividad. Por tal motivo, el tratamiento mejor evaluado fue LATT, a pesar de que una de sus parcelas también presentó una alta mortalidad. La mortalidad debida al efecto del viento se generó por el desraizamiento y quiebre de muchos árboles, que al caer botaron o quebraron otros. En consecuencia, las parcelas con mayores tasas de mortalidad fueron aquellas más expuestas al viento, independientemente de si estaban raleadas o no. Por ejemplo, una parcela testigo ubicada en una cumbre expuesta al viento tuvo una mortalidad de $7 \%$ anual. En cambio, una parcela raleada a 4 metros, ubicada en una hondonada protegida del viento, tuvo una mortalidad de sólo $2 \%$ anual. Es decir, hubo una alta variabilidad entre las parcelas de cada tratamiento.

En el tratamiento LBT25 la mortalidad sólo fue relevante durante los primeros cinco años del ensayo, lo cual está asociado a los cambios estructurales producidos en el renoval por la ejecución del raleo. En el segundo periodo de evaluación la mortalidad disminuyó a 1,8\% anual. Algo similar se observó en los tratamientos HT3 y HT23.

En general, la mortalidad no fue un factor relevante en los ensayos Lenca B y Hueicoya, a pesar de que el tratamiento HT4 fue más fuerte que los tratamientos de raleo de Lenca A, lo cual deja en evidencia la importancia del efecto del viento en este último. No existe una relación directa entre intensidad de raleo y mortalidad, sino más bien entre esta última y la exposición al viento.

La resistencia de los árboles con respecto al viento depende de la firmeza del anclaje (profundidad y área de enraizamiento, tamaño y número de raíces e interconexiones), el tamaño y clase de copa, la relación altura/ diámetro y la elasticidad, resistencia y forma del tronco (Stathers et al. 1994). En el caso de Lenca A todos estos factores se conjugaron para desencadenar la caída masiva de árboles y su correspondiente efecto dominó. El anclaje de los árboles es débil debido a varias razones: a) crecimiento sobre troncos viejos formando raíces tipo zancos, altamente inestables; b) enraizamiento limitado por la presencia de estratos arcillosos a $10-20 \mathrm{~cm}$ de profundidad que limitan el arraigamiento a mayores profundidades; y c) alto grado de interconexión entre los sistemas radicales de los árboles.

Otro aspecto que pudo aumentar la caída de árboles es el tipo de raleo aplicado. Al realizar un raleo por lo bajo, 
dejando individuos dominantes y codominantes y eliminando prácticamente todas las especies acompañantes en el estrato inferior, se aumenta la vulnerabilidad de los árboles con respecto al viento debido a la alta relación altura/diámetro y la ausencia de un sostén lateral dado por los árboles vecinos (Stathers et al. 1994). Los árboles remanentes no habrían generado la resistencia necesaria para soportar los vientos del sector. $\mathrm{Al}$ crecer durante 28 años en el interior de una masa densa y compacta de vegetación, prácticamente no produjeron madera de tracción, lo cual disminuyó su flexibilidad (Pérez 1983). El crecimiento a altas densidades también generó una poda natural fuerte, de modo que las copas de los individuos residuales se concentraban sólo en el dosel superior. Además, la forma elipsoidal de los fustes los hizo más vulnerables, principalmente en el sentido de su diámetro más angosto (Pérez 1983).

Si bien en Lenca B los tratamientos LBT25 y LBTT no tienen diferencias estadísticamente significativas con respecto a volumen, en términos de área basal sí las hubo en favor de LBT25. De todas formas y considerando el mayor tiempo de respuesta requerido por el sitio Lenca $\mathrm{B}$, dadas sus limitaciones de drenaje del suelo, se esperan diferencias significativas a nivel de incremento en volumen para los próximos años. De hecho, durante el periodo 1986-1996 los tratamientos equivalentes en el ensayo Hueicoya no tuvieron diferencias significativas en volumen, pero sí durante el periodo 1986-2002, es decir, con seis años más de crecimiento.

En Hueicoya las tasas de crecimiento en área basal y volumen fueron similares durante los primeros 10 años entre los tratamientos HT4, HT3 y HT23, siendo un poco mayores en los tratamientos HT3 y HT23. Sin embargo, este último tuvo un incremento diametral anual significativamente menor que los tratamientos HT4 y HT3, mientras estuvo raleado a 2 metros. Durante los seis años siguientes las diferencias entre los tratamientos HT4 y HT3 siguieron siendo escasas, sin embargo siempre hubo una mayor productividad en el segundo tratamiento. Estos resultados sugieren que debe buscarse una compensación entre un buen crecimiento en diámetro a nivel de árboles individuales y volumen a nivel de rodal, y ese punto ocurre aproximadamente con el tratamiento HT3 en este caso.

En general, las parcelas raleadas se comportaron de la misma manera, un primer periodo (5-6 años) de mayor crecimiento y un segundo (5-6 años) mucho más bajo, lo cual se debería a una disminución del efecto del raleo. Esto se observa con claridad en las diferencias de crecimiento en área basal y volumen observadas entre el primer periodo de evaluación en los ensayos Lenca A y B (1991-1996), y el segundo periodo 1996-2001. Al respecto, puede ser que la respuesta a los raleos no haya sido fuerte debido a que los renovales se encontraban en una etapa fustal avanzada, lo cual concentró gran parte de sus energías en el crecimiento diametral, ocupando rápidamente los recursos disponibles e incrementando la competencia (Navarro et al. 1999, Nyland 2002).
En general, los mejores resultados se observan en aquellos tratamientos que llevaron la densidad relativa del rodal a un rango entre 25 y $45 \%$ (raleos entre 2 y 3 metros), con un área basal residual entre 20 y $36 \mathrm{~m}^{2} / \mathrm{ha}$. La intensidad de la intervención dependería del sitio, principalmente de la exposición al viento, y de los productos que sea de interés obtener (leña, metro ruma o madera aserrada de alta calidad). Es fundamental evaluar la exposición al viento de renovales de $D$. winteri en un sitio determinado. Los resultados muestran que no es aconsejable intervenir por primera vez un renoval de alta densidad tan drásticamente como lo informado en el presente trabajo. Entre otras consideraciones es recomendable mantener franjas sin intervención en aquellas zonas más expuestas al viento, para proteger las áreas intervenidas. Además, y considerando los resultados obtenidos en Lenca B, es deseable mantener una mayor diversidad de especies después del raleo. Esta medida además aportaría positivamente a la conservación de la biodiversidad de estos bosques.

\section{AGRADECIMIENTOS}

Este ensayo y sus evaluaciones han sido financiados a través del Convenio de la Corporación Nacional Forestal y la Universidad Austral de Chile (CONAF-UACh) "Ecología y Silvicultura de los Bosques Nativos".

\section{REFERENCIAS}

Appel I. 1993. Evaluación de la regeneración en renovales de canelo (Drimys winteri Forst.) sometidos a diferentes niveles de intervención, en la Cordillera de la Costa de la Provincia de Valdivia. Tesis Ingeniero Forestal. Valdivia, Chile. Facultad de Ciencias Forestales, Universidad Austral de Chile. 78 p.

Balharry C. 1984. Estudio de la estructura y composición de los renovales de canelo (Drimys winteri Forst.) en el Fundo Lenca (X Región). Tesis Ingeniero Forestal. Santiago, Chile. Facultad de Ciencias Agrarias y Forestales, Universidad de Chile. 167 p.

Calquín R. 1986. Índices y clases de sitio para canelo (Drimys winteri (FORST)) en la X Región. Tesis Ingeniero Forestal. Santiago, Chile. Facultad de Ciencias Agrarias y Forestales, Universidad de Chile. 125 p.

Chesney L. 1970. Aptitud papelera del canelo (Drimys winteri (Forst)). Tesis Ingeniero Forestal. Santiago, Chile. Facultad de Ciencias Agrarias y Forestales, Universidad de Chile. $108 \mathrm{p}$.

CONAF (Corporación Nacional Forestal, CL), CONAMA (Comisión Nacional del Medio Ambiente, CL), Universidad Austral de Chile, Pontificia Universidad Católica de Chile, Universidad Católica de Temuco. 1999. Catastro y evaluación de recursos vegetacionales nativos de Chile. Informe Décima Región. Santiago, Chile. 137 p.

Corvalán P, L Araya, R Calquín, V Loewe, S Niebuhr. 1987. El canelo: una alternativa de desarrollo para la Décima Región. 
Volumen IV Resultados. Universidad de Chile, Facultad de Ciencias Agrarias y Forestales, Departamento de Manejo de Recursos Forestales. 125 p.

Cuevas E. 1983. Maderas nativas chilenas de interés en el comercio internacional. Santiago, Chile. FAO: DP/CHI/76/003. (Documento de Trabajo $\mathrm{N}^{\circ} 48$ ).

Di Castri F, ER Hajek. 1976. Bioclimatología de Chile. Santiago, Chile. Imprenta-Editorial de la Universidad Católica de Chile. 129 p.

Donoso C. 1993. Bosques Templados de Chile y Argentina. Santiago, Chile. Editorial Universitaria. 483 p.

Donoso PJ, D Soto, RA Bertín. 2007. Size-density relationship in Drimys winteri secondary forest of the Chiloe island, Chile: effect of fisiografy and species composition. Forest Ecology and Management 239: 120-127.

Fuenzalida H. 1965. Clima. In Corporación de Fomento de la Producción ed. Geografía Económica de Chile. Santiago, Chile. Editorial Universitaria. p. 99-152.

Garrido F. 1981. Los sistemas silviculturales aplicables a los bosques nativos chilenos. Santiago, Chile. FAO:DP/CHI/76/003. (Documento de Trabajo No 39).

Gunckel G. 1980. Estudio de desarrollo y rendimiento de renovales de Drimys winteri en el sector de Corral, Cordillera de la Costa, Provincia de Valdivia. Tesis Ingeniero Forestal. Valdivia, Chile. Facultad de Ciencias Forestales, Universidad Austral de Chile. 94 p.

Kelty M. 1992. Comparative productivity of monocultures and mixed-species stands. In Kelty M, B Larson, C Oliver eds. The Ecology and Silviculture of Mixed-Species Stands. Drodrecht, The Netherlands. Kluwer Academic Publishers. p. $125-141$

Millanao D. 1984. Diferenciación genecológica de dos poblaciones de Drimys winteri (Forst) (IX y $\mathrm{X}$ regiones, Chile). Tesis Ingeniero Forestal. Valdivia, Chile. Facultad de Ciencias Forestales, Universidad Austral de Chile. 73 p.

Morales E, J Gayoso, A Ellies, R Gayoso, A Iroumé, R Mc Donald. 1988. Reconocimiento, evaluación y reclasificación de los suelos del sur de Valdivia costa. Informe Universidad Austral de Chile. Valdivia, Chile. 22 p.

Navarro C. 1993. Evaluación de raleos en un renoval de canelo (Drimys winteri (Forst)) en la Cordillera de la Costa de
Valdivia. Tesis Ingeniero Forestal. Valdivia, Chile. Facultad de Ciencias Forestales, Universidad Austral de Chile. 121 p.

Navarro C, C Donoso, V Sandoval. 1999. Los renovales de canelo. In Donoso C, A Lara eds. Silvicultura de los Bosques Nativos de Chile. Santiago, Chile. Editorial Universitaria. p. 341-380.

Niebuhr S. 1988. Determinación de una función de rendimiento para renovales de canelo (Drimys winteri (Forst)) en la Décima Región. Tesis Ingeniero Forestal. Santiago, Chile. Facultad de Ciencias Agrarias y Forestales, Universidad de Chile.

Nyland R. 2002. Silviculture. Concepts and Applications. New York, USA. McGraw-Hill Companies. 633 p.

Oliver CH, B Larson. 1990. Overview of stand development patterns. In Oliver Ch, B Larson eds. Forest Stand Dynamics. New York, USA. John Wiley. 520 p.

Pérez V. 1983. Manual de propiedades físicas y mecánicas de maderas chilenas. Serie Investigación y Desarrollo CONAF-PNUD-FAO. Santiago, Chile (Documento de Trabajo $\mathrm{N}^{\circ}$ 47).

Rivera H, A Rudloff, P Cruz. 2002. Plan de Ordenación de la Reserva Nacional Valdivia. Una visión para el manejo ecológicamente sustentable de los ecosistemas forestales costeros de la X Región de Los Lagos. Proyecto Manejo Sustentable del Bosque Nativo (CONAF/GTZ/DED-KFW). Santiago, Chile. 236 p.

Soto D, PJ Donoso. 2006. Patrones de regeneración en renovales de Drimys winteri en el centro-norte de la Isla de Chiloé: cambios de acuerdo al tamaño y la densidad relativa. Bosque 27(3): 241-249.

Stathers RJ, TP Rollerson, S Mitchell. 1994. Windthrow Handbook for British Columbia Forests. Research Program Working Paper 9401. Victoria, B.C., Canada. Ministry of Forests Research Program. 31 p.

Urzúa D, H Poblete. 1980. Factibilidad técnica de la producción de tableros de partículas utilizando especies que crecen en los terrenos Nadi. Valdivia, Chile. Facultad de Ciencias Forestales, Universidad Austral de Chile (Informe de Convenio $\mathrm{N}^{\mathrm{o}} 29$ ).
Recibido: 14.04 .09

Aceptado: 05.06.09 